\title{
The importance of being vertical - hydrostatic valves and angle of inclination at implantation Christian Sprung* and Claudia Schulze
}

\author{
Address: Neurosurgical Clinic, Charité, Campus Virchow-Klinikum, Universitätsmedizin Berlin, Augustenburger Platz 1, 13353 Berlin, Germany \\ Email: Christian Sprung* - christian.sprung@ charite.de \\ * Corresponding author
}

from 53rd Annual Meeting of the Society for Research into Hydrocephalus and Spina Bifida Belfast, UK. 24-27 June 2009

Published: 27 November 2009

Cerebrospinal Fluid Research 2009, 6(Suppl 2):S19 doi:10.1186/1743-8454-6-S2-S19

This abstract is available from: http://www.cerebrospinalfluidresearch.com/content/6/S2/SI

(c) 2009 Sprung and Schulze; licensee BioMed Central Ltd.

\section{Background}

In recent publications the performance of gravitational valves for shunting hydrocephalus is reported to be influenced significantly by the angle of inclination in sagittal plane at implantation. But there are other well known reasons for under- and over-drainage like etiology, opening pressure of valve, intra-abdominal pressure, size of patient or obesity. Therefore we evaluated the effect of valve inclination in a series of patients treated with retroauricular implantation of proGAV and compared the influence with other possible parameters.

\section{Materials and methods}

In 128 adult patients (130 cases) with various etiologies treated with a V-P-shunt, the angle of inclination of the gravitational unit, part of the proGAV, in relation to the vertical to the German Horizontal was measured on lateral projections of X-rays or scout-views of CT and correlated with primary over- and under-drainage, also taking into account the primary opening-pressure, size and weight of the patient.

\section{Results}

In 78 cases $(60 \%)$ the angle of implantation was in the acceptable range $+/-10^{\circ}$ relative to the ideal vertical position, 29 patients $(22.3 \%)$ revealed a posterior inclination of $>10^{\circ}$ theoretically predisposing to over-drainage and 23 cases $(17.7 \%)$ an anterior sagittal inclination in danger for under-drainage. But of our 20 cases with over-drainage, only four demonstrated a posterior, whereas five had an anterior inclination of $>10^{\circ}$. On the other hand out of our 33 cases with clinical and radiological signs of underdrainage only nine presented with anterior, but 11 with posterior distinct inclination of $>10^{\circ}$. There was also no unequivocal correlation between the occurrence of these "complications", and the opening pressure of the valve.

\section{Conclusion}

The results of our series do not support the critical reports pointing out the necessity of strict vertical implantation. In our opinion the posture and individual position of the head in daily life is more important. Nevertheless, because the function of gravitational valves depends on the inclination relative to the vertical, it should be implanted to ensure horizontal position of the gravitational unit in the lying and vertical in the standing position of the patient as accurate as possible, bringing up the question of superiority of thoracic implantation. 